\title{
Glomerular structural changes in Type 1 (insulin-dependent) diabetes mellitus: causes, consequences, and prevention
}

\author{
R. Østerby \\ Institute of Experimental Clinical Research, Second University Clinic of Internal Medicine and Institute of Pathology, University of Aarhus, \\ Århus, Denmark
}

Summary. Diabetic nephropathy is caused primarily by advanced glomerulopathy, the renal expression of diabetic microangiopathy. With stereological methods a quantitative description of the structural changes is achieved. The glomerulopathy is characterized by an increase in basement membrane material: thickening of the capillary wall and an increase in mesangial volume relative to glomerular volume, comprising increase in matrix. Among groups of patients conformity between renal function stage and structure exists. The parameters measuring glomerulopathy are normal at the onset of diabetes; patients with normoalbuminuria may show slight basement membrane thickening, or normal parameters; the microalbuminuric group shows a measurable, but moderate glomerulopathy; patients with overt nephropathy have advanced lesions; at this stage heterogeneity among glomeruli makes the estimates weaker. Recent data indicate that the changes in peripheral basement membrane and in mesangial matrix develop in concert and both contribute to the early stage of glomerulopathy in patients with microalbuminuria. As to the consequences of the structural changes the mechanism of albuminuria is not clear. It is suggested that the early glomerulopathy entails other structural modifications, including formation of new vessels which may be the site of leakage. The marked deviations in glomerular filtration rate correspond well with estimates of filtration surface area: in the early hyperfunction state it is increased; in advanced nephropathy it is decreased, due to advanced glomerulopathy in conjunction with glomerular occlusion. The diabetic state is the necessary condition for the glomerulopathy. In relating structural changes to presumed contributing causes no supporting evidence of a relationship with glomerular hyperfunction or hypertrophy was observed. The structural parameters may be useful tools in clinical trials aiming at arresting the development of glomerulopathy, and thereby providing a prevention of diabetic nephropathy.

Key words: Diabetic glomerulopathy, basement membrane thickness, mesangium, mesangial matrix, diabetic microangiopathy, diabetic nephropathy, microalbuminuria, stereology.
The first descriptions of glomerular structural changes in patients with diabetes mellitus date back to the last part of the 19th century [1]. In the early 1950s Lundbak saw glomerular capillary changes as an integral part of a generalized affection of the vascular system in patients with long-term diabetes when he coined the concept of a diabetic angiopathy [2].

Kimmelstiel and Wilson's description of the nodular lesions [3] promoted a long series of structural studies of the kidney in diabetes [1]. This area of research is of great clinical relevance since the progressing structural lesions eventually cause terminal renal failure in a substantial proportion of diabetic patients $[4,5]$.

In essence, morphological studies deal with the description of what is observed in tissue sections, with the aim of achieving information that may be useful in the clinical perspective. Therefore, the ultimate goal is to re- late morphology to consequences, i. e. for glomerulopathy, to the derangements in renal function; to presumed causes, in order to learn about pathogenetic factors, and to the effect of therapeutic intervention in an effort to prevent further progression of structural lesions.

In studying the relationship between structure and clinical variables it is necessary that the verbal description of what is observed with the microscope is transformed into figures, as is done in quantitative morphology, or stereo$\operatorname{logy}[6]$. Using stereological methods we can obtain figures for the composition of the tissue as directly observed, expressed as volume fractions, but also estimates of surface and length as well as number of isolated structures, i.e. quantities which are not immediately perceived from the two-dimensional sections. For the description of diabetic microangiopathy the special issue of determination of membrane thickness has been particularly important. 

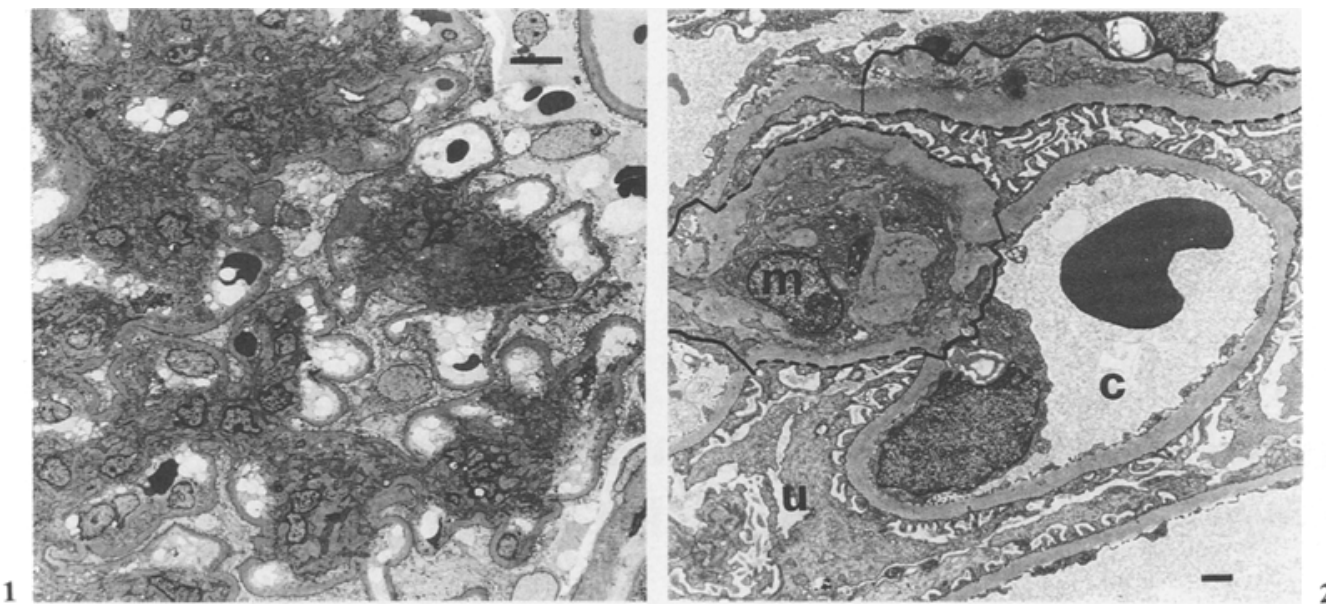

Fig.2. The two mesangial profiles in the micrograph are circled. The dividing line between the peripheral basement membrane and mesangial region is defined by geometrical properties [18]. The stippled lines indicate the mesangial/urinary space, the solid lines the mesangial/capillary space interfaces. Total capillary circumference is the sum of mesangium/capillary interface and peripheral BM. c: capillary space; m: mesangial cell profile; u: urinary space with epithelial cell segments. Bar equals $1 \mu \mathrm{m}$
Fig. 1. Section of a glomerular profile illustrating a rather advanced stage of diabetic glomerulopathy. The patient had overt nephropathy. The solid structures are the mesangial regions which occupy a large proportion of the glomerulus and contain relatively a large amount of matrix. The thickness of the peripheral basement membrane is increased. Bar equals $5 \mu \mathrm{m}$

\section{Glomerular structural changes in diabetes mellitus}

Glomeruli from patients with diabetic nephropathy, i.e. long-term diabetic patients who manifest increasing albumin excretion and decreasing glomerular filtration rate (GFR) have a very characteristic appearance (Fig. 1). The solid spaces in the tuft, the mesangial regions (Fig. 2) occupy a very large fraction of the glomeruli; the capillary walls are thickened due to basement membrane (BM) thickening, and in the mesangial regions the extracellular material, the mesangial matrix occupies a very large fraction of the region. Each of these changes represents a quantitative change of the glomerular composition, and the key word is basement membrane augmentation.

\section{Quantitative morphological methods applied to diabetic glomerulopathy}

The appearance of the characteristic, fairly advanced diabetic glomerulopathy indicates which data are needed for the quantitative description: the thickness of the peripheral basement membrane (PBM), (Fig.2), the fraction of mesangial volume per glomerular volume, $\mathrm{V}_{\mathrm{v}}$ (mes/glom), and the matrix volume as fraction of glomerular volume, $V_{\mathrm{V}}$ (matrix/glom).

Two conditions are critical when obtaining stereological data, one unbiased sampling, which is due at all levels, from the constitution of patient groups to the recording of individual measurements. Second, a precise definition of the structures to be quantitated is necessary (Fig.2). When these requirements are adhered to there are no choices to be made by the observer, apart from designing the most efficient protocol for the sampling, with respect to the structural quantity under study.

After the completion of a stereological study the results are available as figures. The three structural quantities that define the diabetic glomerulopathy are very simple expressions, which may be further combined in a structural index [7], i.e. one figure for the estimate of the severity of glomerulopathy in each individual. But an important matter in the interpretation of the results is the question of the validity of individual figures. Each structural parameter is an estimate of the structural quantitiy in the particular kidney. Unlike many other biological variables related to a particular individual the parameters are obtained in a subsample. The sampling universe being restricted to the biopsy specimen, which constitutes but a very small fraction of the entire kidney, obviously the deviations in estimates from the true values may be larger the more non-homogeneous the renal affection is. Knowledge as to these possible deviations in individual cases is limited. In this context it is important to distinguish the normal from the pathological conditions, and clearly, the situation may be different when different stages in the development of pathological lesions are considered. Some information may be obtained from the variation of data within groups and within individuals. It has been found in the normal situation, that the variation, expressed as e.g. the intra-group coefficient of variation $(\mathrm{CV}=\mathrm{SD} /$ mean $)$, in BM thickness is low, usually below $10 \%$ [8], whereas the variation in the estimates of volume fractions of mesangium and of matrix have been somewhat higher $\sim 20 \%$ [8-10]. The same condition is true for the intra-individual variation, as it was found when comparing two separate biopsies from kidney donors [11]. It is therefore quite difficult to detect early changes in mesangial regions. A systematic study of the sources of this variation in mesangial estimates showed that a very large fraction of the total variation is due to variation within glomeruli [10]. This led to a protocol in which three levels per glomerulus are sampled, spaced by $60 \mu \mathrm{m}$, and the set positioned at a random level within individual glomeruli [12]. Using this sampling protocol the intra-biopsy $\mathrm{CV}$ of mesangial volume fration in a group of diabetic patients with microalbuminuria was found to be as low as 0.12 whereas the corresponding CV in non-diabetic cases, sampling only one level per glome- 


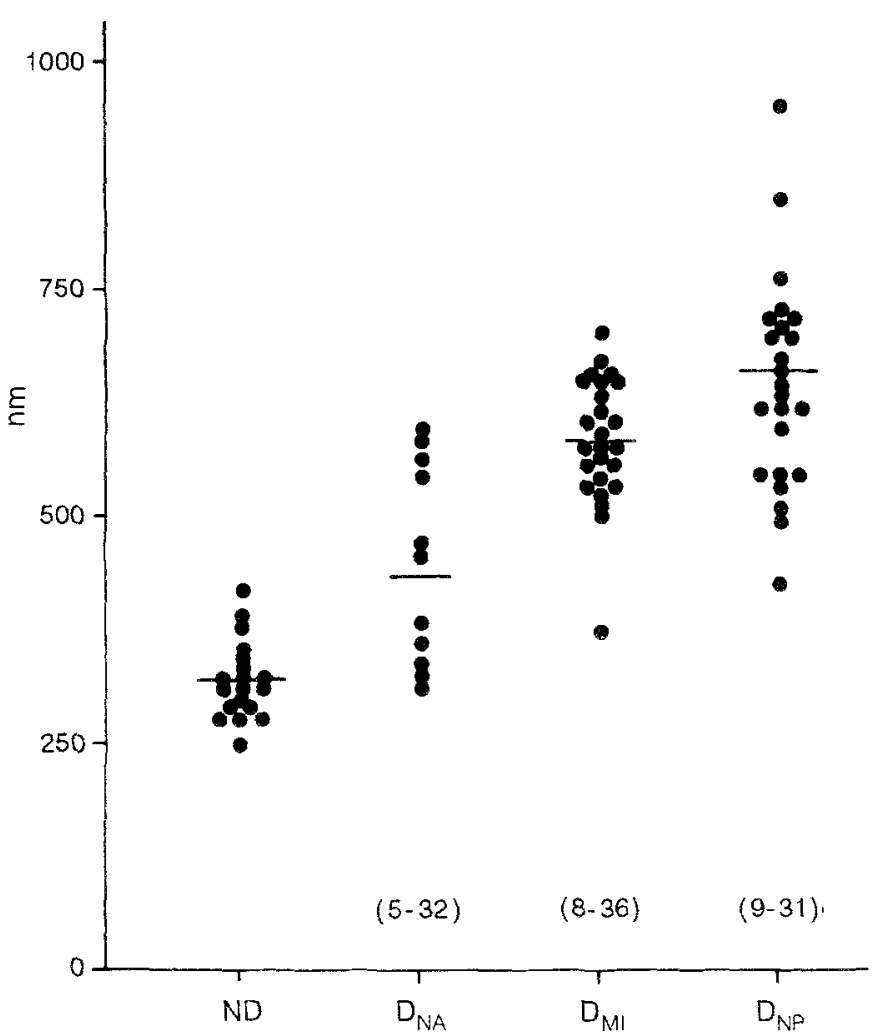

Fig.3. Basement membrane thickness in a group of non-diabetic subjects (ND), diabetic patients with normal albumin excretion $\left(D_{\mathrm{NA}}\right)$, with microalbuminuria $\left(\mathbf{D}_{\mathrm{MI}}\right)$, and with proteinuria (overt nephropathy) $\left(\mathrm{D}_{\mathrm{NP}}\right)$. Figures in parentheses indicate the range of diabetes duration in the respective groups

rulus, was 0.25 (unpublished observation). More extensive sampling, either by increasing the volume under study in each glomerulus, or by increasing the number of glomeruli, seems to be necessary for the detection of early changes in the mesangium.

\section{Diabetic glomerulopathy in patient groups defined by renal function}

Research of renal structure in patients is restricted since renal biopsies can only be obtained under specific conditions. Diabetic groups under study in recent years have been identified by their renal functional state: patients with microalbummuria, i.e. albumin excretion rate (AER) in the range from 15 to $200 \mu \mathrm{g} / \mathrm{min}$ in overnight samples [13], and patients with proteinuria (AER above $200 \mu \mathrm{g} / \mathrm{min}$ ), which is often associated with decreasing GFR, as in patients with overt nephropathy. Further, a limited number of cases with AER in the normal range, i.e. below $15 \mu \mathrm{g} / \mathrm{min}$ have been studied. In individual patients the function stages represent periods of time, since all patients who eventually develop nephropathy have passed the stage of microalbminuria, and they all start with normal albumin excretion. The transition from normo- to microalbuminuria is a clinically very significant sign, because the presence of persistent microalbuminuria indicates a very high risk of progressing to overt nephro-

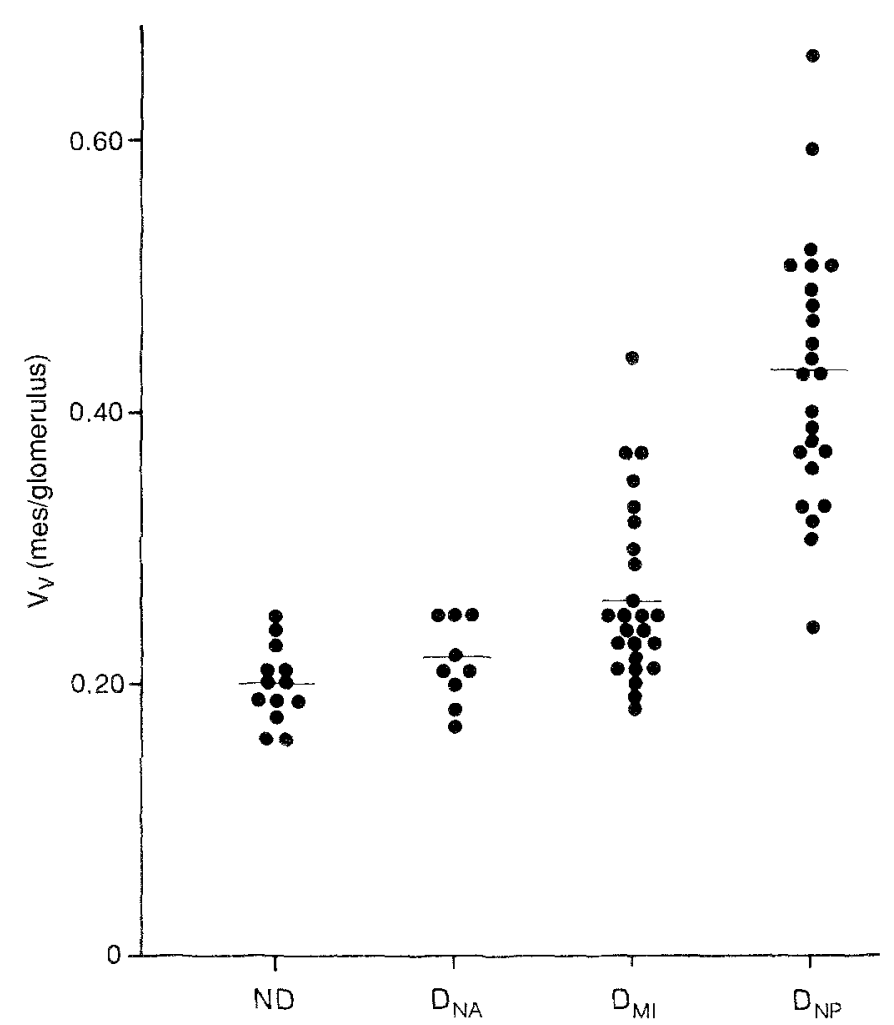

Fig.4. Mesangial space expressed as fraction of total glomerular volume. The glomerular reference space is defined as the minimal, convex, polygon circumscribing the tuft. The groups are defined by renal function state as in Figure 3 . In this figure and in Figure 5 only biopsies studied by level sectioning are included in the $D_{\mathrm{NA}}$ group

pathy [14-16]. The question to morphologists is: what is the cause of this transition? There are no structural data in individual cases followed during this development. Therefore, the approach to this question is to compare glomerular structure in the functionally defined groups.

Earlier, the obtaining of normal kidney tissue comprising control groups was extremely difficult. However, with the introduction of renal transplantation the accessibility of normal tissue - that is donor kidneys at the time of transplantation - has changed this situation. Yet, the problem of demarcating what is "normal" has not been solved [17].

The diabetic glomerulopathy parameters are shown in groups of diabetic patients defined by renal function, together with data from non-diabetic subjects (ND) in Figures $3-5$. The results are pooled data from a number of series studied in our laboratory $\{7,12,18-20$, and unpublished results].

\section{Thickness of the peripheral basement membrane}

The non-diabetic group includes living as well as cadaveric kidney donors, with a very wide age range (up to 68 years), and cases with no information on renal function. The range of BM thickness in this heterogeneous non-diabetic group is wider than found earlier in betterdefined control groups [18]. 


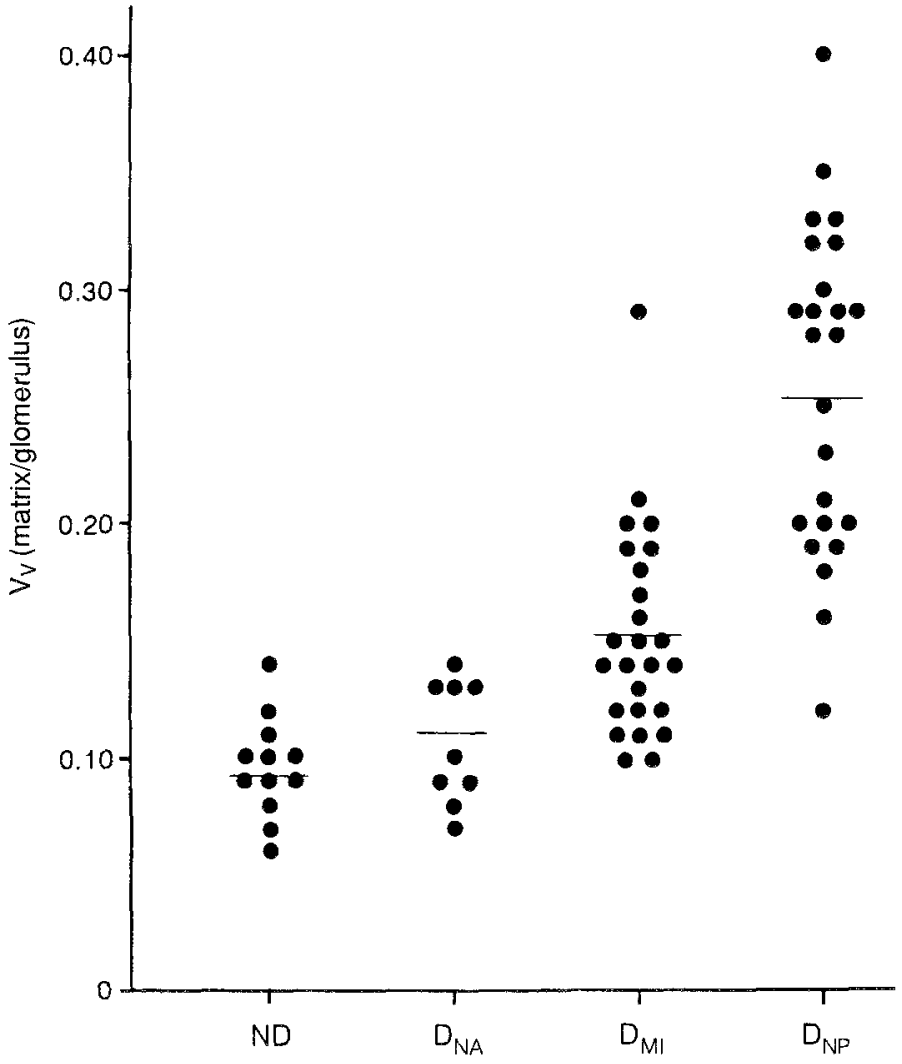

Fig.5. The mesangial matrix expressed as fraction of total glomerular volume. The patient groups are as in Figure 3

The three functionally defined diabetic groups have about the same range of diabetes duration. Clearly, the group characterised by albumin excretion within the normal range $\left(D_{N A}\right)$ holds cases with normal thickness, as well as some with an obviously increased thickness. The group of patients with persistent microalbuminuria $\left(D_{M I}\right)$ had BM thickness completely above the normal range, with the exception of one outlier. It is noteworthy that the further progression, from microalbuminuria to overt nephropathy $\left(\mathrm{D}_{\mathrm{NP}}\right)$ is rather modest.

\section{Mesangial and matrix volume fractions}

Results of estimates of mesangial volume fraction, $\mathrm{V}_{\mathrm{v}}(\mathrm{mes} / \mathrm{glom})$, and of matrix volume fraction, $\mathrm{V}_{\mathrm{v}}$ (matrix/glom) are shown in Figures 4-5. The mesangial volume fraction is entirely within the normal range in group $\mathrm{D}_{\mathrm{NA}}$, and also in a substantial proportion of the $\mathrm{D}_{\mathrm{MI}}$ group. On the other hand, there is a clear increase from the microalbuminuric stage to overt nephropathy.

In conclusion, on a crude scale there is conformity between the functional and structural stages: patients with overt nephropathy have advanced glomerulopathy; patients with microalbuminuria have clearly increased glomerulopathy parameters compared with the group of diabetic patients with normal albumin excretion. Among the latter cases there are signs of early glomerulopathy in terms of BM thickening in some patients, whereas others are within the normal range for all parameters.
With increasing pathology $\left(\mathrm{D}_{\mathrm{MI}} \rightarrow \mathrm{D}_{\mathrm{NP}}\right)$ there is increasing intra-group variation in the parameters (Figs. 3-5). In fact, in patients with overt nephropathy the estimates of glomerulopathy become less representative of the kidney involvement, a fact that should be borne in mind when results in different groups are compared (Figs. 3-5). In the advanced stage nephron heterogeneity may be marked, and the estimates obtained in a given biopsy may be quite imprecise. Furthermore, in this stage several confounding factors may influence glomerular structures as well as renal function, e.g. glomerular occlusion, in combination with large vessel involvement and pronounced interstitial changes. Combining the percentage of glomerular occlusion with glomerulopathy parameters into a structural index a good correlation can be obtained between structure and function when a range of overt nephropathy is considered [7].

\section{Mesangium vs peripheral basement membrane}

The above results raise the question whether the mesangial changes and those in the peripheral basement membrane are separate, independent phenomena. The pattern of development seems to be different, as summarised in Figure 6. Clearly, the thickening of the peripheral BM is the first structural change seen, either because it is the initial lesion, or because our detection limit of the mesangial changes is still too high. Suggestive of a parallel course of development in the peripheral capillary walls and in the mesangium is the finding of a positive correlation between the thickness of the peripheral BM and the mesangial parameters (BM thickness vs mesangial volume fraction: $\left.r=0.66, p<10^{-4}\right)$ in the combined group of normo- and microalbuminuric patients, which spans the range from normal to early glomerulopathy.

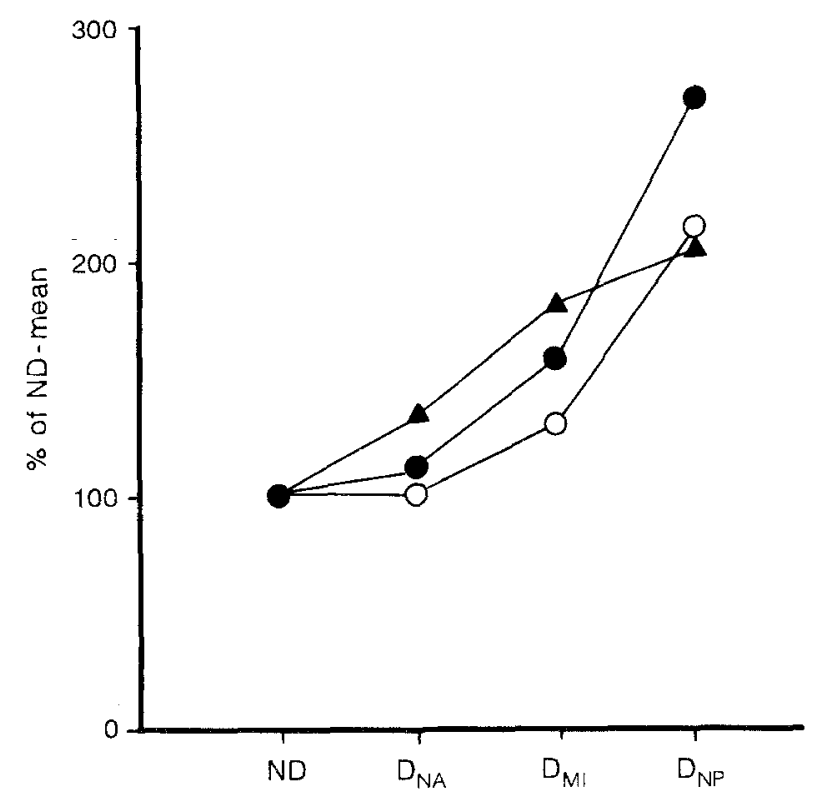

Fig.6. Group mean values expressed as percent of the non-diabetic (ND) mean value. The patient groups are as in Figure 3. The structural parameters are: $\boldsymbol{\Lambda}$, basement membrane thickness; $O$, mesangial volume fraction; , matrix volume fraction 


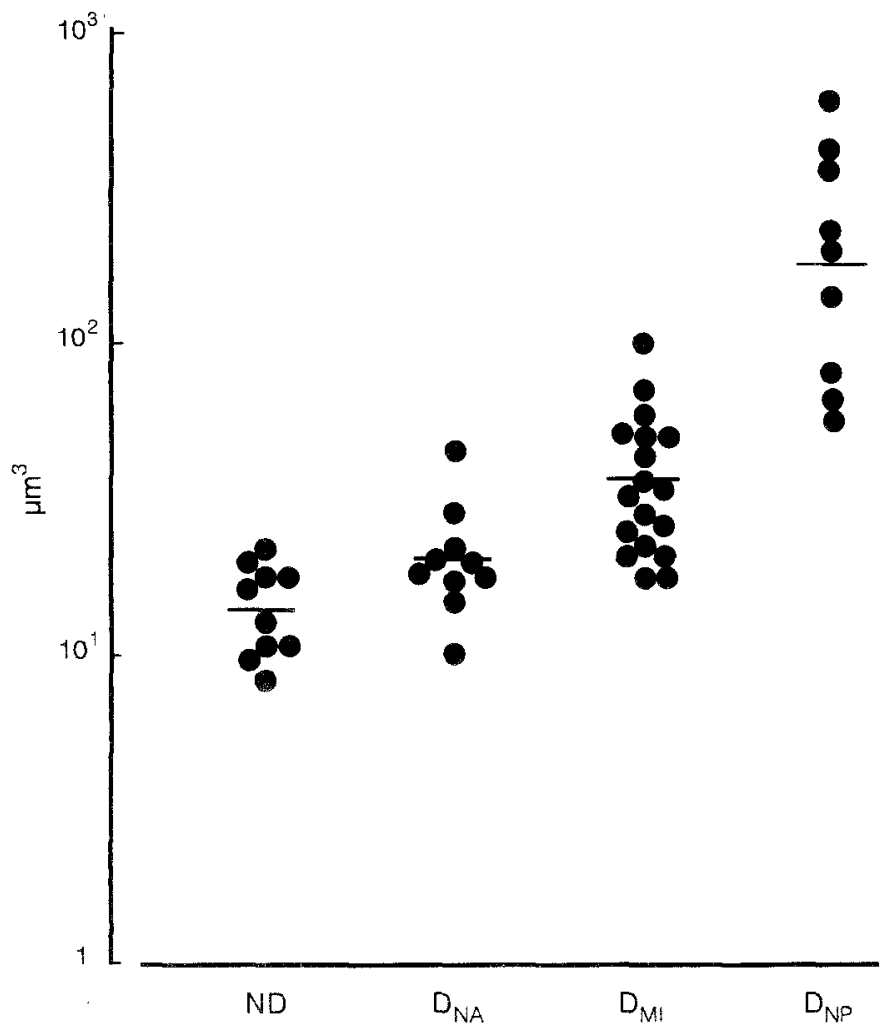

Fig.7. The matrix star volume is shown in the same groups as defined in Figure 3. The star volume is the average of volumes that can be filled in with straight lines (in three-dimensions) from random points within the matrix [21]

Comparison of results in the normo- and microalbuminuric groups indicates that the initial events in the mesangium are related to matrix changes rather than to a "mesangial cell proliferation". The matrix volume fraction, however, is the product of two relatively weak estimators, and alternative expressions of matrix structure were sought.

\section{Matrix star volume}

The so-called "star-volume" for a given structure is the average of volumes that can be filled-in with straight lines from random points within the structure [21]. The estimate is obtained by measuring intercepts through random points within the matrix, with three-dimensional uniform orientation distribution [12]. Matrix star volume in the functionally defined groups is shown in Figure 7. Increase in this parameter in the $\mathrm{D}_{\mathrm{MI}}$ group as compared with the control groups and the $\mathrm{D}_{\mathrm{NA}}$ group is evident, as is also a further increase in patients with overt nephropathy. Increasing star volume reflects a higher degree of confluency and/or convexity of the matrix. This change in matrix shape is tantamount to a higher degree of separation of the individual segments of the mesangial cells, a change that may interfere profoundly with the various functions of the mesangial cells. With the relative scarcity of mesangial cells the cell to cell communication is likely to be impeded, and it may eventually reach a critical point when regulation of mesangial cell functions is abolished. A stage of 'passive' matrix accumulation may then be the final pathway towards glomerular obsolescence.

\section{Matrix "thickness"}

This is a derived structural quantity, expressing the thickness which the matrix would have if it were spread out in an even layer on the urinary surface of mesangial regions [12]. It is calculated as: $\left[\mathrm{V}_{\mathrm{v}}\right.$ (matrix/tuft)]/] $\mathrm{Sv}$ (matrix/ urinary space)].

Since it will incease with a 'pure' enlargement of the mesangium, i. e. without change of mesangial composition, as well as with increasing matrix volume fraction, it is probably a very sensitive parameter of early mesangial changes. Figure 8 shows the clear increase in this matrix parameter in the group of patients with microalbuminuria.

Altogether, the findings point towards changes in peripheral $\mathrm{BM}$ and in matrix taking place in concert in the development of early diabetic glomerulopathy. Therefore, the decisive glomerular change is BM accumulation, as shown in Table 1.

\section{Glomerulopathy vs duration of diabetes}

The structural changes of diabetic glomerulopathy develop slowly over years. The BM accumulation may develop at varying rates during different time periods; but it is unlikely to regress. Therefore, undoubtedly, in the indi-

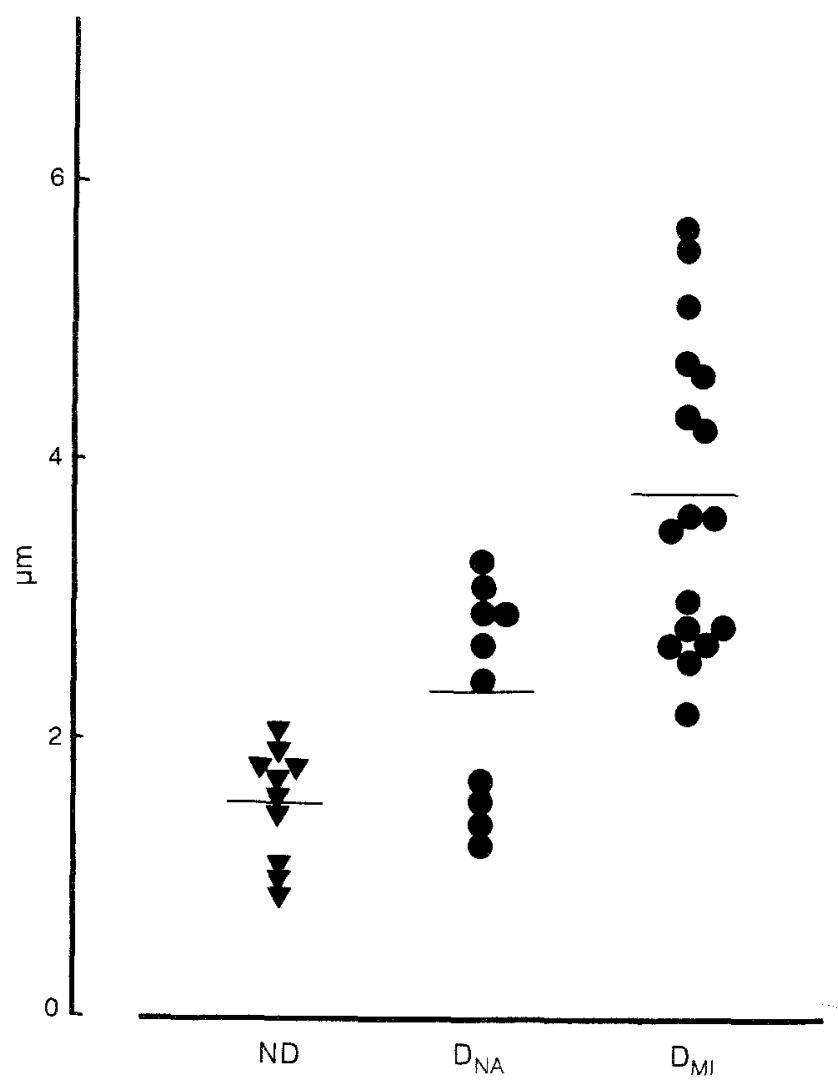

Fig. 8. An arbitrary matrix thickness is shown: the thickness of matrix if spread as an even layer on the mesangial/urinary space interface. It is calculated as the ratio of volume fraction and surface density [12] 
Table 1. Glomerular basement membrane material

\begin{tabular}{|c|c|c|c|c|c|}
\hline \multirow[b]{2}{*}{ Group } & \multirow[b]{2}{*}{$n$} & \multicolumn{2}{|c|}{$\begin{array}{l}\text { Volume of PBM }+ \\
\text { matrix per glomerulus }\end{array}$} & \multicolumn{2}{|c|}{$\begin{array}{l}\mathrm{PBM} / \mathrm{PBM}+ \\
\text { matrix }\end{array}$} \\
\hline & & $\overline{\operatorname{Mean}\left(10^{6} \mu^{3}\right)}$ & $\mathrm{CV}$ & $\overline{\text { Mean }}$ & $\mathrm{CV}$ \\
\hline ND & 8 & 0.45 & 0.33 & 0.38 & 0.38 \\
\hline $\mathrm{D}_{\mathrm{NA}}$ & 9 & 0.52 & 0.23 & 0.42 & 0.08 \\
\hline $\mathrm{D}_{\mathrm{MI}}$ & 17 & 0.79 & 0.29 & 0.40 & 0.24 \\
\hline $\mathrm{D}_{\mathrm{NP}}$ & 14 & 0.99 & 0.34 & 0.19 & 0.51 \\
\hline
\end{tabular}

Total basement membrane volume is the product of volume fraction, estimated by point counting at $\sim 10,000 \times$ magnification, and mean glomerular volume, measured by Cavalieri's principle [21] in plastic embedded tissue. Only in group $\mathrm{D}_{\mathrm{NP}}$ was mean glomerular volume estimated from mean sectional area in paraffin embedded tissue [20]. PBM, peripheral basement membrane; $C V$, coefficient of variation; $N D$, non-diabetic subjects; $D_{\mathrm{NA}}$, diabetic patients with normal albumin excretion; $\mathrm{D}_{\mathrm{MI}}$, diabetic patients with microalbuminuria; $\mathrm{D}_{\mathrm{NP}}$, diabetic patients with proteinuria (overt nephropathy)

vidual patients there is a monotonous, positive correlation between duration of diabetes and the severity of glomerulopathy. Data on this relationship, in particular the extent to which the rate of development may vary over time, are however, almost non-existent. It is a well-known, although unexplained fact, that the duration of diabetes before the appearance of nephropathy varies considerably among patients. Therefore, to look for correlations between duration of diabetes and the severity of glomerulopathy in the groups of patients defined by renal function is hardly meaningful. In particular, when the more advanced stages are considered, the remodelling of glomerular structures that may take place in response to the changes of advanced glomerulopathy influences the parameters. For instance, new vessel formation (see below) may explain the seeming deceleration of BM thickening from the stage of microalbuminuria to overt nephropathy (Fig. 6).

\section{Consequences of structural changes}

The two main functional aspects to consider in the course of diabetic nephropathy are the AER and the GFR. The initially detectable derangement is the increase in AER which, with sensitive methods, is found well in advance of any decline in GFR, i. e. often concurrent with hyperfiltration. The increase in AER is important in terms of heralding the GFR decline which is likely to develop after some time [14-16]. This connection is conceivable, if the albuminuria is a sign of glomerular structural abnormalities which are likely to progress to the stage when they interfere with GFR. On the other hand, the idea that the increased leakiness per se may play a role in the further progression of pathological changes has been put forward [22].

The structure-function relationship has not as yet been clarified concerning an increase in AER, whereas it is rather clear for the GFR.

\section{Albumin excretion vs glomerulopathy}

The combined group of diabetic patients we have studied, with normo- and microalbuminuria, showed a positive correlation between AER and each of the structural pa- rameters, as well as with the overall extent of glomerulopathy expressed as the index: $B M$ thickness $/ 10+\mathrm{V}_{\mathrm{V}}$ (matrix/glom) -100; [log AER vs glomerulopathy index: $r=$ $0.63, n=33, p=0.001]$. Therefore, the data indicate an association between the appearance of increased albumin excretion and the development of diabetic glomerulopathy. But a deeper insight into the mechanism behind the increased leakiness of the filtration barrier is still lacking. The escape of larger particles must indicate the presence of larger pores, so a qualitative change of the barrier must be assumed to have taken place. How it is related to the glomerulopathy is unknown. It is a priori unlikely that the albuminuria should be a direct consequence of current BM thickness or mesangial volume fraction. The albuminuria in the early phase is usually fluctuating from day to day, whereas the structural parameters are obviously invariant within this time scale. The albuminuria is therefore more likely to be related to the developing glomerulopathy in an indirect fashion, which is far from established, but some cautious suggestions can be made.

Intrinsic structure of the peripheral basement membrane. In a series covering a range of albumin excretion, including proteinuria, we estimated the frequency of fluffy intrinsic structure of the peripheral $\mathrm{BM}$, and we found a positive correlation between the extent of this loose, fluffy structure and the level of AER [7]. Areas of loose structure are only observed in some segments, and the suggestion is that the albumin escape takes place in only a small fraction of the capillary surface, corresponding to the physiologists' large pores [23]. However, no explanation for the appearance of such areas in BM exposed to a diabetic milieu for many years is available. Much interest is paid to the possible lack of negative charges [24]. Other changes in the biochemical make-up of the BM material, such as advanced glycosylation, could also be considered [25]. The loosening could be a result of the combined effect of biochemical changes and local haemodynamic conditions related to the altered geometry of the glomerular capillaries caused by local mesangial enlargement.

New vessel formation in diabetic glomeruli. Lobules within the glomerular tuft with capillary structure that deviates markedly from the remainder of the tuft were first observed in cases of quite advanced glomerulopathy [26]. The suggestion was put forward that these areas represent new vessel formation. The capillary walls at these sites vary in thickness with extremely thin areas, alternating with areas with fluffy cushions. They resemble capillaries in immature, developing tufts, and it is easy to imagine, but not necessarily correct, that capillaries of this category may be leaky, in particular when under haemodynamic stress. In our recent series of biopsies from patients with microalbuminuria we have observed similar formations. They may be an expression of a glomerular enlargement taking place at this stage of development. Our data on glomerular volume are still preliminary, but they do indicate larger glomeruli in the group of diabetic patients with microalbuminuria, an observation that correponds with the finding of enlargement of the whole kidney in this patient category [27]. 
Table 2. Ratio of interfaces in the glomerular tuft

\begin{tabular}{|c|c|c|c|c|c|}
\hline \multirow[b]{2}{*}{ Group } & \multirow[b]{2}{*}{$n$} & \multicolumn{2}{|c|}{$\begin{array}{l}\text { PBM/total } \\
\text { capillary surface }\end{array}$} & \multicolumn{2}{|c|}{$\begin{array}{l}\text { [mesangial/urinary space] } \\
\text { [mesangial/capillary space }\end{array}$} \\
\hline & & Mean & $\mathrm{CV}$ & Mean & $\mathrm{CV}$ \\
\hline ND & 12 & 0.72 & 0.07 & 1.01 & 0.20 \\
\hline $\mathrm{D}_{\mathrm{NA}}$ & 9 & 0.69 & 0.05 & 0.85 & 0.19 \\
\hline $\mathrm{D}_{M I}$ & 17 & 0.71 & 0.06 & 0.66 & 0.14 \\
\hline $\mathrm{D}_{\mathrm{NP}}$ & 21 & 0.52 & 0.14 & 0.58 & 0.30 \\
\hline
\end{tabular}

Ratio of surfaces is the ratio of intersections between test lines and the respective boundaries in the micrographs. The countings were done at low magnification electron microscopy $(2,500-5,000 \times$ magnification).

PBM, peripheral basement membrane; $C V$, coefficient of variation; $\mathrm{ND}$, non-diabetic subjects; $\mathrm{D}_{\mathrm{NA}}$, diabetic patients with normal albumin excretion; $D_{\mathrm{MI}}$, diabetic patients with microalbuminuria; $\mathrm{D}_{\mathrm{NP}}$, diabetic patients with proteinuria (overt nephropathy)

In newly formed capillaries the entire thickness of the $\mathrm{BM}$ has been laid down by cells that have been diabetic throughout their life-cycle, and that may influence the biochemical make-up - e.g. the heparan - sulphate proteoglycan.

Modification of surfaces in developing glomerulopathy. Surfaces in the glomerular tuft, i. e. the interfaces between various compartments, may be very important in glomerular function, in particular the flux of solutes from one compartment to the other.

In Table 2 the ratio of various surfaces of the tuft is presented. The capillary surface consists of two subsets, one is the peripheral BM, the other is the mesangial/capillary interface (Fig.2). Only in advanced glomerulopathy is a shift from the normal in the ratio of the two surfaces obvious: the peripheral, or directly filtering part, makes up a smaller fraction of the total capillary surface, i. e. a larger proportion of the blood flows along the mesangial surface. As for the surfaces of the mesangium it is apparent that the group of microalbuminuric patients already has a distribution of surfaces that is different from normal: the entry side to the mesangium, the mesangialcapillary interface, is relatively larger than the exit side towards the urinary space. It is speculated that this shift may favour the accumulation of blood-borne substances within the mesangium, thereby impeding the normal mesangial function of 'cleaning' or 'unclogging' the peripheral filter [28].

The interrelationship between glomerulopathy and microalbuminuria is far from clear. It seems most likely that the diabetic glomerulopathy leads to other structural modifications, e.g. modifications of the normal mesangial architecture induce a glomerular growth with the formation of new vessels, which may be the sites of leakiness.

\section{Glomerular filtration rate vs glomerulopathy}

The structural determinant of the filtration rate is the area of the filtration surface. The term 'filtration surface' is often used for the peripheral BM, although filtration also takes place via the mesangial regions.
Throughout the span of diabetes duration there are remarkable alterations in the GFR, at least in the typical Type 1 (insulin-dependent) diabetic patient. In early diabetes the GFR is increased [29], and it remains so usually into the stage of microalbuminuria. Changes in the size of the surface area of glomerular capillaries may well explain a large part of the functional derangements in the early stages. The same is true concerning the decrease in GFR which occurs at the stage of clinical nephropathy.

Filtration surface in early diabetes. At the onset of diabetes an enlargement of the total area of filtration surface per glomerulus was found [30] in conjunction with glomerular hypertrophy [31]. Studies of patients with diabetes of short duration revealed a close correlation between the size of the filtration surface and the level of GFR [32].

The estimate of filtration surface area per glomerulus includes determination of glomerular volume, a difficult matter when kidney biopsies are considered, due to the limited number of glomeruli available. Information on changes in glomerular dimension over the course of diabetes is therefore scarce and tentative [33, 34]. It may be that the initial glomerular hypertrophy regresses to some extent in parallel with a decrease in kidney size and GFR after the institution of insulin treatment [35]. Such regression is seen in animal experiments after metabolic normalization [36], and it would fit with the finding of glomerular volume in the normal range in diabetic patients with normal renal function [34]. At any rate, it is important to note that glomerular volume is not a fixed measure, but may vary within relatively short periods of time [36-38].

Filtration surface in diabetic nephropathy. The decline in GFR in diabetic nephropathy has been found to correlate with decreasing filtration surface $[33,39]$. This would $a$ priori seem to be a direct consequence of the mesangial expansion. The relationship is, however, not so simple. It has been found that the total filtration surface per open glomerulus in patients with nephropathy is in fact of about the same magnitude as in normal glomeruli $[20,34]$. If other structures remain unchanged, the unquestionable increase in mesangial volume fraction at this stage $[40,41]$ would entail a decrease in filtration surface. The absence of the expected negative correlation between mesangial volume fraction and filtration surface per glomerulus [7] seems to be due to a marked increase in glomerular volume in the patients with nephropathy $[20,34]$. An autopsy series of diabetic patients with overt nephropathy studied by serial sectioning at light microscopy showed that the largest glomeruli were the least affected by diabetic glomerulopathy, which indicated a compensatory enlargement of the best preserved glomeruli [42]. The loss of filtration surface per nephron (i. e. per open + occluded glomeruli) was to a large extent due to glomerular occlusion, which is a prominent phenomenon in patients with advanced glomerulopathy $[20,34,40]$. Glomerular occlusion is the ultimate result of progressive BM accumulation; but also renal macroangiopathy may play a significant role [43]. Thus, the late demise in renal function is a consequence of advanced glomerulopathy, even if the relationship is not straight forward. The results underline the importance of combining 
various structural quantities to obtain the relevant parameter. In these cases the mesangial volume fraction as an isolated figure would not provide functionally meaningful information. Increase in mesangial volume fraction, if totally compensated for by glomerular enlargement, does not result in loss of filtration surface, although obviously the surface density is decreased.

Individual patients may have different capacities to produce the compensatory glomerular growth. Bilous et al. [34] found that the patients who develop nephropathy after a longer duration of diabetes have larger glomeruli than 'fast-track-patients'. What conditions the individual differences in the rate of development is not known. Kidneys with a high degree of heterogeneity in the expression of glomerulopathy among nephrons would seem to have a better capacity to compensate for the progressive glomerulopathy by glomerular growth.

\section{Causes of structural changes in diabetic glomeruli}

Another important aspect of structural studies is to relate structural changes to presumed causes. If we can identify clinical variables that influence the course, we would have indications of relevant targets for therapeutic intervention.

The diabetic state is the necessary condition for the structural changes described above. That the diabetic milieu is sufficient to produce the changes, i. e. independent of any genetic predisposition, is strongly substantiated by studies in experimental diabetes [44]. Further, it is an interesting observation that glomerular cells in vitro produce more BM when they are exposed to high glucose [45, 46]. Still, in addition to these fundamental mechanisms, other conditions that prevail in the diabetic state may play a contributing role.

\section{Glomerular hyperfunction vs glomerulopathy}

In recent years much interest has focused on the glomerular hyperfunction early in diabetes. This functional change precedes the development of glomerulopathy, and the possibility therefore exists that the functional abnormality may affect the glomerular structures adversely. The idea of a harmful effect of glomerular hyperfunction originated from results in animal experiments [47].

In clinical follow-up studies an association was found between the degree of hyperfiltration during standard insulin treatment over the years of diabetes and development of nephropathy $[29,48]$. This finding, however, does not necessarily mean that the hyperfunction per se is involved in the pathogenesis of glomerulopathy. The hyperfunction state is associated with a generally poorer metabolic control [29], thus the glomeruli are exposed to a more abnormal diabetic milieu over time.

In our structural studies of kidney biopsies we found no correlation between BM thickness and GFR in the group of diabetic patients with normoalbuminuria $(r=-0.17)$. In the group with microalbuminuria a negative correlation was found, close to statistical significance, $(r=$
$-0.42, p=0.04)$, i.e. the patients in the group with the lowest GFR, and therefore probably the furthest in the development towards overt nephropathy, were those with the thickest BM. Corresponding results were obtained in both diabetic groups for the relationship between the structural index and GFR: for normoalbuminuric patients GFR vs index: $r=-0.19$; for microalbuminuric patients $r=-0.50, n=21, p=0.02$. It should be stressed that the normoalbuminuric group is probably too small for an absent correlation to be conclusive. Further, in the microalbuminuric group we are confronted with the problem of a non-monotonous course of GFR over the span of diabetes. The results so far gave no support to the proposition of a harmful effect of high GFR.

\section{Glomerular hypertrophy vs glomerulopathy}

Another relevant question is whether the glomerular hypertrophy affects the ensuing development of pathological changes. An association between glomerular hypertrophy and later glomerular lesions has been pointed out in experimental animals as well as in human patients $[49,50]$.

We have studied the intra-biopsy correlation between glomerular volume and glomerulopathy in early stages of diabetes. With serial sectioning for light microscopy combined with ultrastructural studies at a number of levels per glomerulus the glomerulopathy parameters as well as glomerular volume were obtained for individual glomeruli. The variation in size among glomeruli in one biopsy was often marked with a CV of 20-30\%. Intra-biopsy coefficients of correlation showed a random distribution around zero with no preferential occurrence of positive values [10]. Therefore, within a given biopsy the glomerulopathy is not particularly expressed in the largest glomeruli.

We have also followed the early changes in glomerular structures in kidneys transplanted to diabetic patients [38, 51]. Diabetic kidneys showed glomerular enlargement 6 months after transplantation. The early stage of glomerulopathy which was demonstrable 2-3 years after transplantation did not correlate with the early hypertrophy.

A study of the distribution of advanced glomerulopathy in specific zones within the kidney also aimed at elucidating the possible role of glomerular size in the pathogenesis of glomerulopathy [43]. The deep juxtamedullary glomeruli are larger than the superficial corpuscles, and a comparison was made of the severity of glomerulopathy in the deep vs the superficial layers of cortex. No differences were found between the zones.

The results obtained so far have not given any support to the hypothesis that larger glomeruli are more prone to develop glomerulopathy.

\section{Prevention of structural lesions}

The outstanding challenge in the care of diabetic patients is to find strategies which will minimise the development of the vascular complications, amongst them diabetic glo- 
merulopathy. If the progression of glomerulopathy is halted, there will be no nephropathy. The progression of structural changes should preferentially be arrested at as early a time point as possible, before the structural changes lead to secondary changes, setting in motion a cascade of events. Clinical trials are being carried out, especially in patients with microalbuminuria, since this initial stage is one which is clinically detectable. Intervention treatment has been shown to decrease the AER [52]- but whether that is of benefit in terms of abolishing the later decline in GFR is not yet known.

Can we evaluate the effect of intervention treatment by the structural parameters some years earlier than by the functional parameters? This is a question to be answered in the years to come. It is obvious that the glomerular ultrastructure for several reasons in not to be used as a preclinical marker of patients at risk of nephropathy. It might be that we could get reliable expressions in terms of risk by studying BM thickness in normoalbuminuric patients in repeat biopsies. But this is not feasible. Alternatively, in the preclinical state, morphological parameters might be useful to evaluate whether a proposed prevention treatment has any effect in terms of arresting the BM thickening. It seems from our results, that without BM thickening patients will not proceed to the categories of danger: microalbuminuria or proteinuria.

\section{Final remarks}

The present overview has focused quite narrowly on the glomerular ultrastructure. In the analysing process it is necessary, for technical reasons, to take things apart. But it should be emphasized at this point that there is more to the kidney than the glomeruli. Also, what matters to the patient is the overall function of the kidney which depends very much on the intergrated network of various structural compartments. It is one of the challenges to future morphological studies to try and connect changes in one compartment with those in others. The nephron functions as a unique entity. Changes taking place in one segment interfere with other segments. The glomerular function is regulated to a very high degree, by interaction with the rest of the nephron, with other nephrons, with the juxtaglomerular blood vessels, and with the nervous system and hormones. Also the less 'specialised' part of the kidney the interstitium may in fact play a very important role in the integrated outcome of renal function [53].

Big challenges lie ahead. In particular, we are at a time when much more information is being gathered at the level of molecular biology, an area where answers may be found to some of the remaining questions.

Acknowledgements. The results presented in this overview have been obtained in collaboration with many collegues: in Århus, Denmark: K. Lundbæk, G. Gregersen, H.J.Gundersen, A. Hørlyck, J.P. Kroustrup, C. E.Mogensen; Chiba, Japan: K. Hirose; Copenhagen, Denmark: E. Hommel, H.-H. Parving; Gothenburg, Sweden: G. Nyberg, C.Svalander; London, UK: G.C.Viberti, J.D. Walker; Minneapolis, USA: S.M.Mauer, M.W.Steffes; Oslo, Norway: H.-J. Bangstad, K. F. Hanssen. I want to express my sincere gratitude for friendship and fruitful collaboration over the years. Further, the very important support for our studies that has been donated by a great number of funds is gratefully acknowledged.

\section{References}

1. Thomsen $\AA \mathrm{C}$ (1965) The kidney in diabetes mellitus. Munksgaard, Copenhagen

2. Lundbæk K (1953) Long-term diabetes. Munksgaard, Copenhagen

3. Kimmelstiel P, Wilson C (1936) Intercapillary lesions in the glomeruli of the kidney. Am J Pathol 12: 83-105

4. Andersen AR, Sandahl Christiansen J, Andersen JK, Kreiner S, Deckert T (1983) Diabetic nephropathy in Type 1 (insulin-dependent) diabetes: an epidemiological study. Diabetologia 25: 496-501

5. Borch-Johnsen K, Andersen PK, Deckert T (1985) The effect of proteinuria on relative mortality in Type 1 (insulin-dependent) diabetes mellitus. Diabetologia 28: 590-596

6. Gundersen HJG (1980) Stereology - or how figures for spatial shape and content are obtained by observations of structures in sections. Microscopica Acta 83: 409-426

7. Østerby R, Parving H-H, Hommel E, Jørgensen HE, Løkkegaard H (1990) Glomerular structure and function in diabetic nephropathy: from early to advanced stages. Diabetes 39: $1057-$ 1063

8. Østerby $\mathrm{R}$ (1990) Basement membrane morphology in diabetes mellitus. In: Rifkin H, Porte D (eds) Diabetes mellitus, theory and practice, 4th edn. Elsevier, New York Amsterdam London, pp 220-233

9. Steffes MW, Barbosa J, Basgen JM, Sutherland DER, Najarian JS, Mauer SM (1983) Quantitative glomerular morphology of the normal human kidney. Lab Invest 49: 82-86

10. Østerby R, Bangstad H-J, Hanssen KF et al. (1990) Stereological studies of early phases of diabetic glomerulopathy. Diabetologia 33 [Suppl]: A147 (Abstract)

11. Østerby R, Nyberg G, Andersson G, Frisk B (1988) Glomerular structural quantities in baseline biopsies from cadaveric donor kidney pairs. APMIS [Suppl] 4: 130-136

12. Walker JD, Close CF, Jones SL et al. (1992) Glomerular structure in Type-1 (insulin-dependent) diabetic patients with normoand microalbuminuria. Kidney Int 41: 741-748

13. Mogensen CE, Chachati A, Christensen CK et al. (1985-86) Microalbuminuria: an early marker of renal involvement in diabetes. Uremia Invest 9: 85-96

14. Parving H-H, Oxenbøll B, Svendsen PAa, Christiansen JS, Andersen AR (1982) Early detection of patients at risk of developing diabetic nephropathy: a longitudinal study of urinary albumin excretion. Acta Endocrinol (Copenh) 100: 550-555

15. Viberti GC, Hill RD, Jarrett RJ, Argyropoulos A, Mahmud U, Keen H (1982) Microalbuminuria as a predictor of clinical nephropathy in insulin-dependent diabetes mellitus. Lancet I: 1430-1432

16. Mogensen CE, Christensen CK (1984) Predicting diabetic nephropathy in insulin-dependent patients. N Engl J Med 311: $89-93$

17. Nyberg G, Hedman L, Blohmé I, Svalander C (1992) Morphologic findings in baseline kidney biopsies from living related donors. Transplant Proc 24: 355

18. Østerby R (1975) Early phases in the development of diabetic glomerulopathy. A quantitative electron microscopic study. Acta Med Scand [suppl] 574 (Thesis)

19. Østerby R, Andersen AJ, Gundersen HJG, Jørgensen HE, Mogensen CE, Parving H-H (1984) Quantitative studies of glomerular ultrastructure in type I diabetics with incipient nephropathy. Diab Neph 3: 95-100

20. Østerby R, Gundersen HJG, Nyberg G, Aurell M (1987) Advanced diabetic glomerulopathy. Quantitative structural characterization of non-occluded glomeruli. Diabetes 36: 612-619 
21. Gunderson HJG, Bendtsen TF, Korbo L et al. (1988) Some new, simple and efficient stereological methods and their use in pathological research and diagnosis. APMIS 96: 379-394

22. Remuzzi G, Bertani T (1990) Is glomerulosclerosis a consequence of altered glomerular permeability to macromolecules? Kidney Int 38: 384-394

23. Tomlanovich S, Deen WM, Jones HW III, Schwartz HC, Myers BD (1987) Functional nature of glomerular injury in progessive diabetic glomerulopathy. Diabetes 36: 556-565

24. Deckert T, Feldt-Rasmussen B, Borch-Johnsen K, Jensen T, Kofoed-Enevoldsen A (1989) Albuminuria reflects widespread vascular damage. The Steno hypothesis. Diabetologia 32:219-226

25. Brownlee M, Cerami A, Vlassara H (1988) Advanced glycosylation end products in tissue and the biochemical basis of diabetic complications. N Engl J Med 318: 1315-1321

26. Østerby R, Nyberg G (1987) New vessel formation in the renal corpuscles in advanced diabetic glomerulopathy. J Diab Compl 1: $122-127$

27. Feldt-Rasmussen B, Hegedüs L, Mathiesen ER, Deckert T (1991) Kidney volume in Type 1 (insulin-dependent) diabetic patients with normal or increased urinary albumin excretion: effect of long-term improved metabolic control. Scand J Clin Lab Invest 51:31-36

28. Kanwar YS (1984) Biology of disease. Biophysiology of glomerular filtration and proteinuria. Lab Invest 51: 7-21

29. Mogensen CE (1989) Hyperfiltration, hypertension, and diabetic nephropathy in IDDM patients. Diab Nutr Metab 2: 227-244

30. Kroustrup JP, Gundersen HJG, Østerby R (1977) Glomerular size and structure in diabetes mellitus. III. Early enlargement of the capillary surface. Diabetologia 13: 207-210

31. Østerby R, Gundersen HJG (1975) Glomerular size and structure in diabetes mellitus. I. Early abnormalities. Diabetologia 11: 225-229

32. Hirose K, Tsuchida H, Østerby R, Gundersen HJG (1980) A strong correlation between glomerular filtration rate and filtration surface in diabetic kidney hyperfunction. Lab Invest 43: 434437

33. Ellis EN, Steffes MW, Goetz FC, Sutherland DER, Mauer SM (1986) Glomerular filtration surface in type I diabetes mellitus. Kidney Int 29: 889-894

34. Bilous RW, Mauer SW, Sutherland DER, Steffes MW (1989) Mean glomerular volume and rate of development of diabetic nephropathy. Diabetes 38: 1142-1147

35. Mogensen CE, Østerby R, Gundersen HJG (1979) Early functional and morphological vascular renal consequences of the diabetic state. Diabetologia 17:71-76

36. Gøtzsche O, Gundersen HJG, Østerby R (1981) Irreversibility of glomerular basement membrane accumulation despite reversibility of renal hypertrophy with islet transplantation in early experimental diabetes. Diabetes 30: 481-485

37. Seyer-Hansen K (1983) Renal hypertrophy in experimental diabetes mellitus. Kidney Int 23: 643-646

38. Østerby R, Nyberg G, Karlberg I, Svalander C (1992) Glomerular volume in kidneys transplanted into diabetic and nondiabetic patients. Diab Med 9:144-149
39. Østerby R, Parving HH, Nyberg G et al. (1988) A strong correlation between glomerular filtration rate and filtration surface in diabetic nephropathy. Diabetologia 31: 265-270

40. Mauer SM, Steffes MW, Ellis EN, Sutherland DER, Brown DM, Goetz FC (1984) Structural-functional relationships in diabetic nephropathy. J Clin Invest 74: 1143-1155

41. Steffes MW, Østerby R, Chavers B, Mauer SM (1989) Mesangial expansion as a central mechanism for loss of kidney function in diabetic patients. Diabetes 38: 1077-1081

42. Gundersen HJG, Østerby R (1977) Glomerular size and structure in diabetes mellitus. II. Late abnormalities. Diabetologia 13: $43-48$

43. Hørlyck A, Gundersen HJG, Østerby R (1986) The cortical distribution pattern of diabetic glomerulopathy. Diabetologia 29: $146-150$

44. Østerby R (1988) Glomerular structural abnormalities in early and late stages of experimental diabetes: models for diabetic nephropathy? In: Shafrir E, Renold AE (eds) Frontiers in diabetes research: lessons from animal diabetes II, John Libbey, London, Paris pp 522-527

45. Bernstein J, Cheng F, Roszka J (1987) Increased glucose increases glomerular basement membrane in metanephric culture. Pediatr Nephrol 1: 3-8

46. Haneda M, Kikkawa R, Horida N et al. (1991) Glucose enhances type IV collagen production in cultured rat glomerular mesangial cells. Diabetologia 34: 198-200

47. Hostetter TH, Rennke HG, Brenner NM (1982) The case for intrarenal hypertension in the initiation and progression of diabetic and other glomerulopathies. Am J Med 72: 375-380

48. Rudberg S, Persson B, Dahlquist G (1992) Increased glomerular filtration rate predicts diabetic nephropathy - Results from an 8 -year prospective study. Kidney Int (in press)

49. Yoshida Y, Fogo A, Ichikawa I (1989) Glomerular hemodynamic changes vs. hypertrophy in experimental glomerular sclerosis. Kidney Int 35: 654-660

50. Klahr S, Schreiner G, Ichikawa I (1988) The progression of renal disease. N Engl J Med 318: 1657-1666

51. Østerby R, Nyberg G, Hedman L, Karlberg I, Persson H, Svalander C (1991) Kidney transplantation in Type 1 (insulin-dependent) diabetic patients. Early glomerulopathy. Diabetologia 34: 668-674

52. Mathiesen ER, Hommel E, Giese J, Parving H-H (1991) Efficacy of captopril in postponing nephropathy in normotensive insulin dependent diabetic patients with microalbuminuria. Br Med J 303: $81-87$

53. Pinter GG, Atkins JL (1991) Role of postglomerular microvessels in pathophysiology of diabetic nephropathy. Assessment and hypothesis. Diabetes 40: 791-795

Dr. R. Østerby

Electron Microscopy Laboratory

Institute of Pathology

Kommunehospitalet

DK-8000 Århus C

Denmark 